\title{
Clinical Analysis on the Use of the Photon Therapeutic Apparatus Combined with Peal Burn Ointment in Treatment for Breast Cancer Patients
}

\author{
Chun-Ni FAN, Ning LIU, Guang SUN* \\ Breast Surgery Department of China-Japan Union Hospital of Jilin University, \\ No.169,Xiantai Street Changchun Jilin Province, 130021. \\ ${ }^{*}$ Corresponding author
}

Keywords: Photon therapeutic apparatus, Peal Burn Ointment, Skin flap necrosis.

\begin{abstract}
Objective: To investigate Carnation-87C photon therapeutic apparatus combined with peal burn ointment in the clinical effect of breast cancer patients after operation with unhealed incision of skin flap necrosis. Method: The treatment groups in the conventional treatment supplemented by photon therapeutic apparatus combined with peal burn ointment, the control group using route dressing change, two groups of patients with other treatments are the same. The two groups were treated for 4 weeks, to observe fresh wound granulation tissue growth and healing time. Results: Two groups of patients after operation with unhealed incision of skin flap necrosis, fresh wound granulation tissue growth and healing time were statistically significant $(\mathrm{P}<0.05)$. Conclusion: The photon therapeutic apparatus combined with peal burn ointment in treatment for breast cancer patients after operation with unhealed incision of skin flap necrosis has a promoting effect. The combined treatment is worth in clinical application.
\end{abstract}

\section{Introduction}

Since June 2012,Carnation-87C photon therapeutic instrument (Shenzhen Pumen science and Technology Co., Ltd. production) combined with pearl burn ointment (Changchun burns hospital) were used in 42 cases of breast cancer postoperative skin flap necrosis and wound healing in patients of our department. and achieved definitive curative effect.

\section{Clinical Data}

\section{General Information}

The patients we selected from June, 2012 to June, 2015 in China-Japan Union Hospital of Jilin university. These patients suffer with breast cancer and have a postoperative wound skin flap necrosis for a long period (more than 15 days). All patients are available with inclusion criteria, exclusion criteria, excluding standard. By applying the method of random number table to 42 cases of breast cancer patients in the group of number, they are divided into 22 cases in treatment group and 20 cases of control group. The treatment group used Carnation-87C photon therapeutic instrument combined with pearl burn ointment. All patients we chose were female, taken the breast cancer modified radical mastectomy, aged 43-70 (52.49 \pm 10.37$)$ years old. Course of skin flap necrosis was not healing among 15 to $47(21.3 \pm 4.53)$ days. The average wound area was $16.43 \pm 19.86 \mathrm{~cm} 2.13$ tumors on the left breast, and 9 cases on the right breast. The control group used route dressing change. In this group, we chose female patients who 
undergo the breast cancer modified radical mastectomy, aged 41-68 (52.46 \pm 7.32$)$. Course of skin flap necrosis was not healing among 35 to 64 (37.64 \pm 8.43$)$ days. The average wound area was $16.41 \pm 18.54 \mathrm{~cm} 2.6$ tumors on the left breast, and 14 cases on the right breast. Those groups of cases such as gender, age, course of the disease, trauma based indexes $(\mathrm{P}>0.05)$, showed no statistical difference. Correlation analysis form is shown in Table 1.

Table 1. The statistics correlation analysis form with age, treatment days and wound area shows showed no statistical difference $(\mathrm{P}>0.05)$.

\begin{tabular}{|c|c|c|c|c|}
\hline Group & Case number & Age(years) & Treatment days & Wound area \\
\hline Treatment & 22 & $52.49 \pm 10.37$ & $21.3 \pm 4.53$ & $16.43 \pm 19.86$ \\
\hline Control & 20 & $52.46 \pm 7.32$ & $37.64 \pm 8.43$ & $16.41 \pm 18.54$ \\
\hline
\end{tabular}

\section{Inclusion Criteria}

The patient diagnosed with breast cancer by pathology examination. The breast cancer patients have a postoperative wound skin flap necrosis not healing for a long period after taken the breast cancer modified radical mastectomy treatment.

\section{Exclusion Criteria}

Serious infection or autoimmune disease.Allergic to peal burn ointment .Such basic disorders as severe liver, kidney, heart, cerebrovascular, etc.

\section{Treatment and Observation}

\section{Treatment}

The treatment group uses the photon therapeutic apparatus combined with peal burn ointment.After disinfection of the surrounding skin by iodine disinfection tampons, we wash the wound by physiological saline repeatedly, and completely remove the necrotic tissue to normal tissue. Then we use the photon therapeutic apparatus, light source from the wound for $10-15 \mathrm{~cm}$, irradiation time is $10-15$ minutes each time, once a day.After that,we put peal burn ointment to topical wound, outsourcing gauze to absorb wound drainage.Give appropriate pressure, make peal burn ointment can closely integrated with wounds. Dressing change once a day. Observe fresh wound granulation tissue growth and healing status. The control group uses route dressing change, with iodine disinfection disinfection tampon sealed after the surrounding skin, using the scissors to cut off the wound necrotic tissue and physiological saline cotton ball wiping wound purulent secretion off, outsourcing sterile gauze to absorb wound drainage.Give appropriate pressure, make sterile gauze closely integrated with wounds. Dressing change once a day. Observe fresh wound granulation tissue growth and healing status.Two groups of patients with other treatments are the same.

\section{Observe Wound Healing Status and Count the Healing Time}

Healed: wounds healed completely, without scar or scar less than $1 / 2$ of the wound; Effection: wound decreased $75 \%$ or a lot of scar formation.Improved: the wound to reduce $25 \%$.Invalid: narrowing of less than $25 \%$ of the wound.

Between the two groups, the wound is still unhealed after 4 weeks treatment, we continue the same treatment and statistics the time when the wound heals completely. 


\section{Statistical Processing}

SPSS17.0 statistical software is applied, measurement data to $\mathrm{x}+\mathrm{s}$ said, using $\mathrm{t}$ test analysis;count data using chi-square test, $\mathrm{P}<0.05$ for differences is statistical significance.

\section{Results}

After we compare the two groups of patients clinical curative effect after symptomatic treatment. The results shows: in treatment group, there are 22 patients, 18 cases show healed $(81 \%)$,only 1 case shows invalid( $0.45 \%)$. Healing time is $15-47(21.3 \pm 4.53)$ days. At the control group, there are 20 patients, among them, 12 cases show healed (60\%), 3 case show invalid(15\%). Healing time is 35-64(37.6 \pm 8.43$)$ day.Two groups of patients were statistical significance $(\mathrm{p}<0.05)$.

Table2. After we compare the two groups of patients' clinical curative effect after symptomatic treatment, we found that the treatment group compared with control group, with statistical significance $(\mathrm{p}<0.05)$.

\begin{tabular}{|c|c|c|c|c|c|c|c|}
\hline Group & $\begin{array}{c}\text { Case } \\
\text { number }\end{array}$ & Healed & Effection & Improved & Invalid & $\begin{array}{c}\text { Recovery } \\
\text { Rate }\end{array}$ & $\begin{array}{c}\text { Healing } \\
\text { time }\end{array}$ \\
\hline Treatment & 22 & 18 & 2 & 1 & 1 & 81 & $21.3 \pm 4.53$ \\
\hline Control & 20 & 12 & 4 & 1 & 3 & 60 & $37.6 \pm 8.43$ \\
\hline
\end{tabular}

\section{Discussion}

\section{The Reason of Skin Flap Get Necrosis After Breast Cancer Surgery}

In our country, breast cancer has become the most common cancer which often occurred in Female. Surgical treatment is one of the main treatment methods of breast cancer. In current clinical application, there are five operation method most often widely using: breast cancer radical mastectomy, breast enlargement radical prostatectomy, breast cancer modified radical mastectomy, breast total mastectomy, breast-conserving surgery. But each operation method can not completely avoid the occurrence of postoperative complications, flap necrosis is one of the most common complications after breast cancer treatment.Skin flap necrosis after breast cancer influence subsequent treatment effect, not only extending the period living in hospital, but also increasing the economic burden. The choice of incision is not reasonable.Intraoperative incision keep thin skin flap.There is too much tension between the skin flaps when suture the incision. Intraoperative use of high frequency electricity knife. Postoperative subcutaneous drainage is not liquidity. At present, the treatment of skin flap necrosis after breast cancer is to enhance the body's immunity, completely remove local necrosis tissue, keep the incision drainage unobstructed, apply antibiotics to control infection, strengthen local microcirculation, promote the regeneration of connective tissue.It is important to prevent the occurrence of skin flap necrosis after breast cancer surgery.Reasonable selection and design of incision, free and protection flap during surgery are needed. Avoid to use high frequency electricity knife more frequently can reduce the possibility of postoperative subcutaneous effusion. Set drainage tube under the chest and axillary skin respectively can also adequate drainage. Appropriate bandage compression to offer a better pressure upon the incision is also needed. In a word, those are the keys to the prevention and treatment of skin flap necrosis after breast cancer surgery. 


\section{The Characteristics of the Photon Therapeutic Apparatus}

Carnation-87C photon therapeutic apparatus is a new type of treatment, the operation is simple and convenient, no adverse effects on the human body.It can cooperate with drug treatment to promote wound healing, without exception. Data shows that: red light irradiation can promote wound healing and has antiinfection effect, with the visible light emission wavelength of $600 \sim 700 \mathrm{~nm}$, and the best conditions of biological tissue absorption spectrum. Red light can increase the enzyme activity of mitochondria catalase, the decomposition of ATP, the synthesis of protein and glycogen through the photochemical activity in living cells to greatly promote cell metabolism and promote wound repair. In addition, the red light can promote the phagocytosis of white blood cells, its strong penetrability can dilate vessels of deep tissue, strengthen blood flow, and promote the absorption of inflammation, so its anti-inflammatory and anti infection effect are objectively effective. Red light irradiation can also decrease the concentration of 5-HT in local tissue to analgesic . In summary, red light irradiation has the effect of local anti-inflammatory, detumescence, analgesia, which can significantly shorten the healing time to improve hospital quality of care.

\section{The Characteristics and Use of Pearl Burn Cream}

Pearl burn ointment contains pearl, honeysuckle, angelica, rehmannia root, etc., is a kind of compound medicine preparations. It has effect of blood stasis detumescence, detoxification, nourishing muscle, promoting granulation and epithelial tissue growth and vascularization. Those ability can participate in the mechanism of wound healing. In patients with pearl burn ointment, we can observe the remain skin paddle expanding rapidly, to a certain extent, it can replace the skin grafting. Breast cancer postoperative delayed healing is a thorny issue, surgical repair effect is not ideal. Using pearl burn ointment based on the characteristics of its functions, to heal the wounds, obtains better results. Put peal burn ointment to topical wound, outsourcing gauze to absorb wound drainage.Give appropriate pressure, make peal burn ointment closely integrated with wounds. That method can enhance peal burn ointment's ability to remove saprophytic muscle, anti-inflammatory analgesic, and promote the role of epithelial cell growth. Peal burn ointment used for wound can fill gaps, adhesion vascular tissue, exert its anti-inflammatory, promote the collagen of the effect of cell growth, and promote the body cell regeneration. 42 patients has no obvious side effects in the process of using peal burn ointment. 

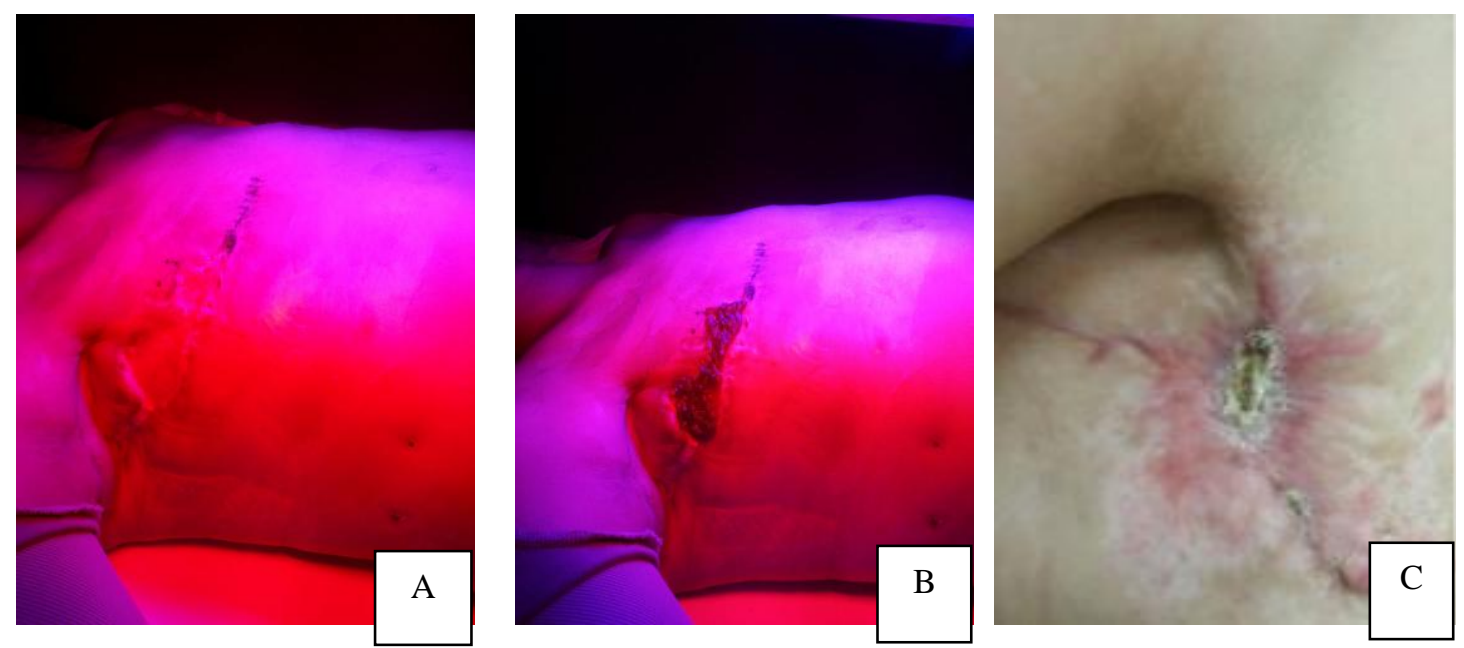

Figure 1. Headings 15 days after breast surgery. There is a $10.52 \times 3.24 \mathrm{~cm} 2$ skin flap necrosis appear on this patient right chest.A: The photon therapeutic apparatus was working.B:Peal burn ointment was put to the topical wound. C:The incision got healed after 4 weeks by using he photon the rapeutic apparatus combined with peal burn ointment.

\section{References}

[1] YU Xue-ming, LI Juan, Clinical observation of the photon therapeutic apparatus in preventing its complications after flap surgery. Chinese Journal of Aesthetic Medicine, 2015, 20:1008-6455.

[2] W. Strunk Jr., E.B. White, The Elements of Style, third ed., Macmillan, New York, 1979.

[3] CHEN Bi-xiu, Clinical analysis of adjuvant therapy of the photon therapeutic apparatus to II ${ }^{\circ}$ burn. Practical Journal of Clinical Medicine.2010, 4:131-132.

[4] Xing Shu-xia, Clinical observation on the application of Pearl burn ointment in treating burn wounds. Chinese Community Doctors.2010, 29(12):127-128

[5] Yang Yu-xin,Jia Xu-feng.Carnation-33 photon therapy combined with Kangfuxin Liquid in treatment of skin ulcer. Zhejiang Clinical Medical Journal.2010, 8(12):856-857.

[6] Li E. The clinical application of photon therapeutic apparatus in anal fistula surgery. Chinese Journal of Integrated Traditional and Western Medicine.2010 (7):256-257.

[7] Su Rong, Li Min-min. Observation on the efficacy of red light irradiation combined with Chinese herbal medicine in the treatment of postoperative wound infections. Clinical Journal of Anhui Traditional Chinese Medicne.2002, 10 (14):370-371.

[8] Carlson GW, BostwickJ, Styblo TM,etal. Skinsparingmastectomy, Oncologicandre constructive consideration [J].AnnSurg, 1997, 225(5):570. 